\title{
Can narrow-band imaging of Peyer's patches predict the recurrence of ulcerative colitis?
}

\author{
Yoon Jae Kim \\ Division of Gastroenterology, Department of Internal Medicine, Gachon University Gil Medical Center, Incheon, Korea
}

\section{Article: Narrow-band imaging with magnifying endoscopy for Peyer's patches is useful in predicting the recurrence of remissive patients with ulcerative colitis (Intest Res 2016;14:314-321)}

Endoscopic evaluation is very important for managing the treatment of patients with UC. Narrow-band imaging (NBI; Olympus, Tokyo, Japan) is an optical endoscopic technique that provides a clearer image of the mucosal layer's microvascular structure than a routine endoscopy using standard visible light can provide. Because the intramucosal vascular structure can be observed using NBI without applying dye, NBI has mainly been used to diagnose gastrointestinal neoplasm. However, clinical use of NBI for diagnosing UC is uncommon to date, and NBI imaging in UC patients has been used to detect dysplasia in surveillance.

Peyer's patches are aggregated lymphoid follicles surrounded by the follicle-associated epithelium, which contains M cells; they are usually found in the lowest portion of the small intestine, the ileum. Peyer's patches can transfer luminal antigens and bacteria, and can be considered the immunity gate of the intestine.

In the current issue, Hiyama et al. ${ }^{1}$ revealed the importance of Peyer's patches for UC patients. The authors observed Peyer's patches in the terminal ileums of UC patients using NBI with magnifying endoscopy and categorized the morphology into two groups, the low type and the high type, based on a "villi index." The duration of time before clinical relapse was shorter in remissive UC patients with high

Received September 1, 2016. Revised September 6, 2016. Accepted September 7, 2016.

Correspondence to Yoon Jae Kim, Division of Gastroenterology, Department of Internal Medicine, Gachon University Gil Medical Center, 21 Namdongdaero 774 beon-gil, Namdong-gu, Incheon 21565, Korea. Tel: +82-32-4603778, Fax: +82-32-460-3408, E-mail: yoonmed@gachon.ac.kr

Financial support: None. Conflict of interest: None. type Peyer's patches than that in those with low type Peyer's patches. However, the types of Peyer's patches were not associated with the extent of disease involvements, clinical activity, degree of inflammation on endoscopy, or concomitant use of medication.

This study revealed several interesting points. It revealed the relationship between small bowel morphological findings of Peyer's patches and the clinical course of UC, which usually involves the colonic mucosa.

Limited attention has been paid to Peyer's patches in relation to the pathogenesis of UC. The importance of Peyer's patches is better known in regard to CD than UC. ${ }^{2,3}$ Peyer's patches are located on the terminal ileum, while UC generally involves the colon and rectum. For this reason, the role of Peyer's patches in the pathogenesis of UC has rarely been evaluated. However, chronic inflammation from UC can sometimes involve the terminal ileum, which is called backwash colitis. For this reason, we supposed that Peyer's patches may contribute to the development of UC.

The course of UC is unpredictable and repeated relapses of UC can lead to cumulative colonic damage. Continuous clinical follow-ups and UC activity assessments are required to plan a therapeutic strategy. Furthermore, it is very important for doctors to have the precise information that can be used to predict the disease course. Monitoring of the disease course of UC is based on a summation of clinical activity, endoscopic images, radiologic images, serological markers, and fecal markers. There are several variables that may indicate aggressive UC, such as raised levels of perinuclear anti-neutrophil cytoplasmic antibodies (p-ANCA), deep ulcerations in the colonic mucosa, lack of mucosal healing after induc-

\footnotetext{
๑ Copyright 2016. Korean Association for the Study of Intestinal Diseases. All rights reserved.

This is an Open Access article distributed under the terms of the Creative Commons Attribution Non-Commercial License (http://creativecommons.org/licenses/by-nc/4.0)

which permits unrestricted non-commercial use, distribution, and reproduction in any medium, provided the original work is properly cited.
} 
tion of clinical remission, development of primary sclerosing cholangitis, pancolitis, and the patient being younger in age $(<40)^{4,5}$

In endoscopic findings, the extent of colon involvement at diagnosis predicts the need for immunomodulators or colectomy. However, pancolitis does not elevate the risk of relapse. ${ }^{6,7}$ Lack of mucosal healing after steroid treatment was also a risk factor of a severe disease course of UC.

Peyer's patches were not correlated with the current severity of UC. Both the degree of clinical activity and the severity of endoscopic disease were not connected with the type of Peyer's patches in the terminal ileum as examined using NBI with magnifying endoscopy. Because of this, we supposed that disease susceptibility and the extent of UC do not have any relationship to the type of a patient's Peyer's patches. However, Hiyama et al. ${ }^{1}$ emphasized that the high type of Peyer's patches predicts the disease course of remitted UC. The cumulative clinical relapse rates at 12 and 24 months after initial endoscopy with NBI was $17 \%$ and $25 \%$ for patients with low type Peyer's patches and $54 \%$ and $73 \%$ for patients with high type, respectively.

There are some limitations to the present study. The definition of "remissive patient" used in this study was a patient with a partial Mayo score $\leq 2$, and the Mayo endoscopic subscore of remissive patients was 0 in 23 patients, 1 in 26 patients, and 2 in three patients. Considering this, the definitive Mayo scores of remissive patients in this study were relatively high. The Mayo scores of some patients may exceed 2 or 3. A stricter definition of remission may be required for the evaluation of "remissive patients." Colonoscopy remains the gold standard for diagnostic work-up in UC patients. However, this investigation method is expensive, invasive, and laborious. NBI with magnifying endoscopy is a more expensive and time-consuming procedure. In the current issue, NBI images of Peyer's patches were very useful for predicting the recurrence of UC, but had limitations, such as cost and risk, in comparison to other methods, such as fecal calprotectin.

Dye-less chromoendoscopy techniques such as NBI, flexible spectral imaging color enhancement (FICE; Fujifilm, Tokyo, Japan), i-scan (Pentax, Tokyo, Japan), and Storz Professional Image Enhancement System (SPIES; Storz, Tuttlingen, Germany), and the use of various dye-based endoscopy techniques using indigo carmine, Lugol solution, cresyl violet, toluidine blue, or methylene blue were attempted to evaluate UC., ${ }^{8,9}$ However, these traditional dyes have been mainly used to detect colorectal neoplasia for surveillance purposes. Recently, several new endoscopic strategies for di- agnosis of UC have been attempted, such as high-definition and optical magnification endoscopy, confocal laser endomicroscopy, molecular imaging, and endocytoscopy. For example, Neumann et al. ${ }^{10}$ reported that confocal laser endomicroscopy and histopathologic grading of disease activity had a concordance rate of $100 \%$. Also, inflammatory status of colonic mucosa and development of cancer have been successfully studied using molecular imaging, even in humans. More trials with such a recent technological method for evaluating the inflammatory status of UC patients could allow individual predictions for the disease course of each patient. The current issue is pioneering and informative for clinicians in this respect.

\section{REFERENCES}

1. Hiyama S, Iijimal H, Kawai S, et al. Narrow-band imaging with magnifying endoscopy for Peyer's patches is useful in predicting the recurrence of remissive patients with ulcerative colitis. Intest Res 2016;14:314-321.

2. Kim MH. Protein phosphatase 1 activation and alternative splicing of Bcl-X and Mcl-1 by EGCG + ibuprofen. J Cell Biochem 2008;104:1491-1499.

3. Shikuwa S, Isomoto H, Mizuta Y, Suematsu T, Ito M, Kohno S. Magnifying videoendoscopic findings of Peyer's patches in the terminal ileum of Crohn's disease. Gut 2007;56:894-895.

4. Ali RA. The positive influences of increasing age at diagnosis of inflammatory bowel disease on disease prognostication in Asian perspective. Intest Res 2015;13:4-5.

5. Yarur AJ, Strobel SG, Deshpande AR, Abreu MT. Predictors of aggressive inflammatory bowel disease. Gastroenterol Hepatol (N Y) 2011;7:652-659.

6. Lau A, Chande N, Ponich T, Gregor JC. Predictive factors associated with immunosuppressive agent use in ulcerative colitis: a case-control study. Aliment Pharmacol Ther 2008;28:606-613.

7. Henriksen M, Jahnsen J, Lygren I, et al. Ulcerative colitis and clinical course: results of a 5-year population-based follow-up study (the IBSEN study). Inflamm Bowel Dis 2006;12:543-550.

8. Lee HS. Detection of polyps including adenomas in the ascending colon by cap-assisted chromocolonoscopy with indigo carmine. Intest Res 2014;12:3-4.

9. Mönkemüller K, Fry LC, Zimmermann L, Mania A, Zabielski M, Jovanovic I. Advanced endoscopic imaging methods for colon neoplasia. Dig Dis 2010;28:629-640.

10. Neumann H, Vieth M, Neurath MF, Atreya R. Endocytoscopy allows accurate in vivo differentiation of mucosal inflammatory cells in IBD: a pilot study. Inflamm Bowel Dis 2013;19:356-362. 